OPEN ACCESS

Edited by:

Thomas W. Weickert, University of New South Wales, Australia

Reviewed by:

Thomas Whitford University of New South Wales, Australia

Maria Angelique Di Biase, Melbourne Neuropsychiatry Centre, Australia

*Correspondence: Marcus Kaiser m.kaiser@ncl.ac.uk:

Peter J. Uhlhaas peter.uhlhaas@glasgow.ac.uk

tSharmili Edwin Thanarajah and

Cheol E. Han contributed equally to this work.

FShared senior authorship.

Specialty section: This article was submitted to Schizophrenia,

a section of the journal

Frontiers in Psychiatry

Received: 21 December 2015 Accepted: 10 June 2016 Published: 30 June 2016

Citation:

Edwin Thanarajah S, Han CE, Rotarska-Jagiela A, Singer W, Deichmann R, Maurer K, Kaiser M and Uhlhaas PJ (2016) Abnormal

Connectional Fingerprint

in Schizophrenia: A Nove

Network Analysis of Diffusion

Tensor Imaging Data.

Front. Psychiatry 7:114 doi: 10.3389/fpsyt.2016.00114

\section{Abnormal Connectional Fingerprint in Schizophrenia: A Novel Network Analysis of Diffusion Tensor Imaging Data}

\author{
Sharmili Edwin Thanarajah ${ }^{1,2,3 \dagger}$, Cheol E. Han ${ }^{4,5,6 \dagger}$, Anna Rotarska-Jagiela ${ }^{2}$, Wolf Singer ${ }^{2,7,8}$, \\ Ralf Deichmann ${ }^{9}$, Konrad Maurer ${ }^{10}$, Marcus Kaiser ${ }^{6,11,12 * \neq}$ and Peter J. Uhlhaas ${ }^{2,13 * f}$ \\ 'Department of Neurology, University Hospital of Cologne, Cologne, Germany, ${ }^{2}$ Department of Neurophysiology, Max-Planck \\ Institute for Brain Research, Frankfurt am Main, Germany, ${ }^{3}$ Max-Planck Institute for Metabolism Research, Cologne, \\ Germany, ${ }^{4}$ Department of Electronics and Information Engineering, Korea University, Sejong, South Korea, ${ }^{5}$ Department of \\ Bio-Convergence Engineering, Korea University, Seoul, South Korea, ${ }^{6}$ Department of Brain and Cognitive Sciences, Seoul \\ National University, Seoul, South Korea, ${ }^{7}$ Ernst-Strüngmann Institut, Frankfurt am Main, Germany, ${ }^{8}$ Frankfurt Institute of \\ Advanced Studies, Goethe University Frankfurt am Main, Frankfurt am Main, Germany, ${ }^{9}$ Brain Imaging Centre, Goethe \\ University Frankfurt am Main, Frankfurt am Main, Germany, ${ }^{10}$ Department of Psychiatry, Psychosomatics and Psychotherapy, \\ Goethe University Frankfurt am Main, Frankfurt am Main, Germany, ${ }^{11}$ Interdisciplinary Computing and Complex BioSystems \\ (ICOS) Research, School of Computing Science, Newcastle University, Newcastle, UK, ${ }^{12}$ Institute of Neuroscience, \\ Newcastle University, Newcastle, UK, ${ }^{13}$ Institute of Neuroscience and Psychology, University of Glasgow, Glasgow, UK
}

The graph theoretical analysis of structural magnetic resonance imaging (MRI) data has received a great deal of interest in recent years to characterize the organizational principles of brain networks and their alterations in psychiatric disorders, such as schizophrenia. However, the characterization of networks in clinical populations can be challenging, since the comparison of connectivity between groups is influenced by several factors, such as the overall number of connections and the structural abnormalities of the seed regions. To overcome these limitations, the current study employed the whole-brain analysis of connectional fingerprints in diffusion tensor imaging data obtained at $3 \mathrm{~T}$ of chronic schizophrenia patients $(n=16)$ and healthy, age-matched control participants $(n=17)$. Probabilistic tractography was performed to quantify the connectivity of 110 brain areas. The connectional fingerprint of a brain area represents the set of relative connection probabilities to all its target areas and is, hence, less affected by overall white and gray matter changes than absolute connectivity measures. After detecting brain regions with abnormal connectional fingerprints through similarity measures, we tested each of its relative connection probability between groups. We found altered connectional fingerprints in schizophrenia patients consistent with a dysconnectivity syndrome. While the medial frontal gyrus showed only reduced connectivity, the connectional fingerprints of the inferior frontal gyrus and the putamen mainly contained

\footnotetext{
Abbreviations: Acc, nucleus accumbens; Amy, amygdala; Caud, nucleus caudatus; COpC, central opercular cortex; FOC, frontal orbital cortex; FopC, frontal operculum cortex; FP, frontal pole; IFG.po, inferior frontal gyrus pars opercularis; IFG.pt, inferior frontal gyrus, pars trigonum; InsC, insular cortex; MFG, medial frontal gyrus; Pal, pallidum; ParaciG, paracingulate gyrus; PostcG, Postcentral Gyrus; PrecG, precentral gyrus; Put, putamen; SFG, superior frontal gyrus; SupplMC, supplementary motor cortex; Thal, thalamus; TP, temporal pole, and $\mathrm{r}$ and $\mathrm{l}$ denoted the right hemisphere and the left hemisphere, respectively (see Table S1 in Supplementary Material).
} 
relatively increased connection probabilities to areas in the frontal, limbic, and subcortical areas. These findings are in line with previous studies that reported abnormalities in striatal-frontal circuits in the pathophysiology of schizophrenia, highlighting the potential utility of connectional fingerprints for the analysis of anatomical networks in the disorder.

Keywords: schizophrenia, diffusion tensor imaging, graph theory, connectional fingerprint, neuroinformatics

\section{INTRODUCTION}

Recent graph theoretical studies characterize the human brain as a complex network of distinct areas that communicate with each other on different spatial and temporal scales (1). Diffusion tensor imaging (DTI) data and tractography algorithms facilitated the investigation of structural brain networks $(2-4)$ and revealed a brain connectome that is optimized to save connection lengths and maximize communication efficiency (1). In addition, recent studies have highlighted the possibility that psychiatric disorders, such as schizophrenia, are characterized by alterations in the organization of functional and anatomical networks (5-7).

Schizophrenia is a complex psychiatric disorder characterized by positive symptoms, such as hallucinations and delusions, as well as negative symptoms and cognitive dysfunctions. There is emerging evidence that these disturbances are associated with a complex pattern of anatomical and functional connectivity anomalies rather than with impairments of isolated cortical and subcortical structures $(8,9)$. This is consistent with graph theoretical analyses of anatomical networks that have indicated a widespread disturbance of several local network characteristics, such as clustering coefficient and path length (10-12), while the overall network topology is preserved.

To detect connectivity changes in the network topology in schizophrenia, previous diffusion-weighted imaging studies $(13,14)$ performed an edge-by-edge comparison of "absolute" sample numbers (absolute connectivity) traced between pairs of brain areas through tractography algorithms and reported reduced cortico-cortical connectivity in several brain regions $(12,13,15)$. However, several functional imaging studies reported both hypo- and hyperconnected areas in schizophrenia compared to healthy controls (13). In particular, emerging evidence suggest frontal hyperconnectivity in schizophrenia patients during rest $(16,17)$ and during working memory $(18)$.

The analysis of absolute connectivity estimates may not be sufficient to characterize aberrant anatomical networks in schizophrenia because they do not account for overall white matter (WM) and gray matter (GM) reductions reported in the disorder (19-21). Hence, we would like to suggest that the analysis of connectional fingerprints in schizophrenia could circumvent some problems associated with absolute connectivity estimates as connectional fingerprints are less affected from the WM and GM reduction than absolute connectivity (22). The connectional fingerprint of a brain area represents the set of connection probabilities to all its target areas $(23,24)$. Specifically, the connection to each target area is quantified by the number of fiber samples and normalized by the total number of fiber samples propagated from that seed area to all its target areas. Hence, the connectional fingerprint provides a unique set of connection probabilities for each brain region (23) that is independent of overall WM reduction and differences in the seed areas (22). Recently, the connectional fingerprint was employed for analyses of functional connectivity (25).

Recent diffusion tensor imaging studies in healthy participants facilitated the investigation of connectional fingerprints to distinguish functionally different subunits in the thalamus (24), in the medial frontal cortex (26), in the geniculate bodies (27), in the lateral frontal cortex $(28,29)$, and in the lateral prefrontal cortex of humans (30) and macaques (22). Brain regions defined through their connectional fingerprint corresponded to parcelations based on anatomical characteristics, including patterns of gyri and sulci (30) and localization based on functional magnetic resonance imaging (fMRI) data, suggesting a possible link between the connectional fingerprint and the function of a brain area $(29,31)$.

In the current study, we employed connectional fingerprints to provide a novel insight into organizational network changes in schizophrenia. The analysis of connectional fingerprint has so far been applied only to specific brain areas $(24,26-29,31)$ and only in healthy participants $(32,33)$. Thus, to our knowledge, the current study is the first whole-brain investigation of connectional fingerprint in a clinical sample. Specifically, we hypothesized that connectional fingerprints in frontal and subcortical areas will be abnormal in the line with the notion of a disconnection syndrome (34). To examine this question, we first detected brain regions whose overall connectional fingerprint differed between groups through similarity analysis and then tested for correlations with clinical symptoms.

\section{MATERIALS AND METHODS}

\section{Participants}

We recruited 16 medicated schizophrenia patients who met the DSM-IV criteria for schizophrenia from the Department of Psychiatry, Johann Wolfgang Goethe University, Frankfurt. We also recruited 17 age- and gender-matched healthy controls from the local community. All patients were on atypical neuroleptics at the time of testing. DSM-IV diagnosis for schizophrenia was confirmed by a trained psychologist with the SCID-interview for DSM-IV-R. Exclusion criteria were for both schizophrenia patients and controls: (1) a neurological disorder, (2) alcohol, nicotine, or substance dependence within the last month, or (3) structural abnormalities in the T1 MR image. After being given a complete description of the study, each participant provided written informed consent. The study was approved by the ethical committee of the Goethe University Frankfurt. Participants were assessed with the Edinburgh Handedness Inventory (35), 
the Brief Assessment of Cognition in Schizophrenia (BACS) battery (36), and the Mehrfach-Wortwahl-Test (37), which is a measure of premorbid, verbal intelligence. Current psychopathology of schizophrenia patients was assessed with the Positive and Negative Syndrome Scale (PANSS) (38) and symptoms were grouped into five factors according to the model of Lindenmayer et al. (39), including the components "positive," "negative," "cognitive," "excitement," and "depression." In addition, we assessed patients for the "disorganization factor" that comprises the items conceptual disorganization, poor attention, and inappropriate affect (40).

\section{Data Acquisition}

MR-data were acquired on a 3-T scanner (Siemens Trio, Erlangen, Germany) at the Brain Imaging Centre (BIC), Frankfurt am Main, Germany. The imaging protocol included high-resolution T1-weighted anatomical MRI and diffusion tensor MRI. The scanner's body coil was used for RF transmission and an eight-channel head coil for signal reception. Head motion was minimized by the use of tightly padded clamps. The T1-weighted anatomical imaging protocol had the following parameters: threedimensional (3D) MPRAGE, sagittal slices, field of view $(\mathrm{FoV})=$ $256 \mathrm{~mm} \times 256 \mathrm{~mm} \times 176 \mathrm{~mm}$, matrix size $256 \times 256 \times 176$, isotropic resolution of $1 \mathrm{~mm}, \mathrm{TR} / \mathrm{TE} / \mathrm{TI}=2250 \mathrm{~ms} / 2.6 \mathrm{~ms} / 900 \mathrm{~ms}$, $\mathrm{FA}=9^{\circ}$, bandwidth $=200 \mathrm{~Hz} /$ pixel. The DTI protocol was chosen as follows: $2 \mathrm{D}$ slice selective spin echo EPI sequence with diffusion encoding, FoV $=192 \mathrm{~mm} \times 192 \mathrm{~mm}$, matrix size $96 \times 96,60$ axial slices with a thickness of $2 \mathrm{~mm}$, no interslice gap, isotropic resolution of $2 \mathrm{~mm}, \mathrm{TR} / \mathrm{TE}=8200 \mathrm{~ms} / 99 \mathrm{~ms}$, $\mathrm{FA}=90^{\circ}$, bandwidth $=1302 \mathrm{~Hz} /$ pixel, echo spacing $=0.85 \mathrm{~ms}$. For DTI analysis, 60 diffusion encoding directions with a $b$-value of $1000 \mathrm{~s} / \mathrm{mm}^{2}$ were covered, and 10 baseline images without diffusion weighting were acquired. We recorded DTI three times for each subject to improve the signal-to-noise ratio with a scanning time of $36 \mathrm{~min}$.

\section{Data Preprocessing}

A brain network consists of nodes that are predefined anatomical regions and edges, which reflect the relationships between any pair of two nodes. To define the nodes, we employed the HarvardOxford Probabilistic MRI atlas developed by FMRIB Oxford and the Harvard Centre for Morphometric Analysis that was predefined on the MNI template based on anatomical landmarks (http://www.cma.mgh.harvard.edu/fsl_atlas.html). We included 48 cortical and 7 selected subcortical areas (thalamus, nucleus caudatus, putamen, pallidum, amygdala, nucleus accumbens, and hippocampus) for each hemisphere (Table S1 in Supplementary Material for the list of nodes and their abbreviation). Using FSL tools from the FMRIB software library (41), we skull-stripped DTI-images without diffusion encoding and a T1-weighted image $(42,43)$. For each participant, we obtained coregistration between these two images, and non-linear registration between a skull-stripped T1-weighted image and the MNI template, using FNIRT (42). Furthermore, we transformed the selected regions of interest defined on the MNI template into the diffusion-weighted images of individuals to ROIs for probabilistic tracking. We note that we visually checked all procedures of skull-stripping, coregistration between each T1 image and its corresponding DTI, and registration between the Harvard-Oxford atlas and diffusionweighted images.

To quantify edges, we performed probabilistic tractography (44). Probabilistic tractography has several advantages over deterministic tractography (45), in particular in the detection of tracts among crossing fibers (46) as well as the reconstruction of fibers also in areas of low anisotropy (47). Moreover, its test-retest reliability is robust $(48,49)$. Before further processing the diffusion tensor images, we serialized three diffusion tensor images and gradient vectors for each subject; thus, a collated image of each subject has 30 b0-images and 180 diffusion images (50). Then, we corrected effects of head motion and eddy currents, registering images with diffusion encodings to the image without diffusion encoding of the first scan; we adequately rotated the gradient vectors after the correction. Subsequently, we performed local modeling of probabilistic diffusion parameters (bedpostX) (44) over the skull-stripped diffusion-weighted images. Then, we conducted probabilistic tracking in DTI space using the classification target tool in ProbtrackX2 (FSL) with 5000 samples/voxel and 110 registered ROIs based on the Harvard-Oxford atlas as explained above. The overall pipeline, including preprocessing, was shown in Figure 1.

\section{Network Construction and Connectional Fingerprint}

Each brain region has the unique set of connection probabilities to all its target areas, which is called its "connectional fingerprint" (23). The connectional fingerprint of each brain area is defined by its target areas and the connection probabilities to these target areas (44). With probabilistic tractography, we detected the number of fiber samples of each seed voxel that reached each of the 109 target areas. The number of fiber samples that reached a certain target area divided by the total number of samples propagated from a voxel represented the connection probability of the seed voxel to a specific target area. We extended this procedure to all voxels in the seed area and averaged their connection probability. Hence, the connectional fingerprint of the node $i\left(w^{i}\right)$ includes the connection probability to a node $j$ that, was defined as:

$$
w_{j}^{i}=\frac{1}{n^{i}} \sum_{q} \frac{c_{j q}^{i}}{\sum_{k} c_{k q}^{i}}
$$

where $c_{j q}^{i}$ represents the number of fiber samples from a voxel $q$ of the node $i$ to the target node $j$, and $n^{i}$ represents the number of seed voxels in the node $i$. The connection probability of the voxel $q\left(c_{j q}^{i} / \Sigma_{k} c_{k q}^{i}\right)$ was averaged for all voxels in the node $i$.

It is important to note that the connectional fingerprint is a node-specific characteristic. Since it is acquired through nodal normalization, $w_{j}^{i}$ is not equal to $w_{i}^{j}$. $w_{j}^{i}$ is the connection probability of node $i$ that takes into account all connections from node $i$, while $w_{i}^{j}$ is normalized for all connections from node $j$. As a simple example, when the total number of fiber samples propagated from the node $j$ is bigger than the one from the node $i$, connection probability $w_{i}^{j}$ would be smaller than $w_{j}^{i}$ (see text 1 in Supplementary Material). 


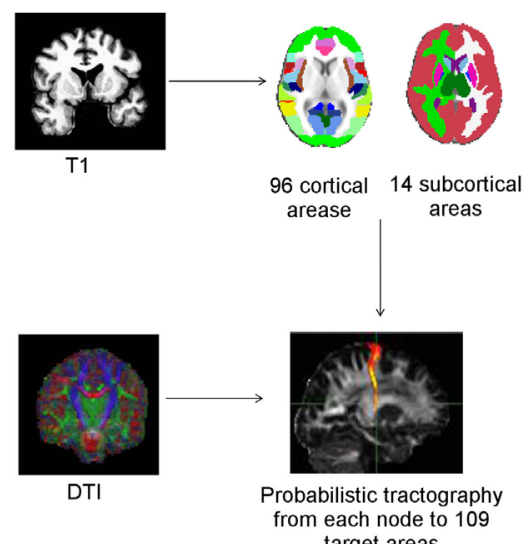

target areas node

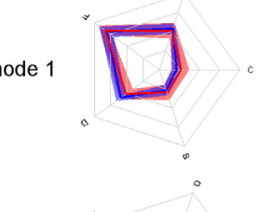

node 2

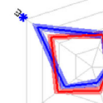

$(2$

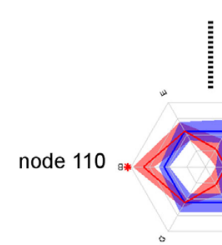

Preprocessing

\begin{tabular}{|cc}
\multicolumn{1}{c}{ Hierarchical comparison } \\
\hline $\begin{array}{l}\text { Step I } \\
\text { Node by Node comparison } \\
\text { Projection pattern similarity }\end{array}$ & Step II \\
Edge by edge comparison \\
Connection probability
\end{tabular}

FIGURE 1 | Overview of data processing and suggested graph theoretical analysis. Left panel: data preprocessing: anatomical data was parcellated into 96 cortical and 14 subcortical regions using FSL (Harvard-Oxford probabilistic atlas, Table S1 in Supplementary Material). Through probabilistic tractography, the fiber samples were detected voxel-wise from each seed area to the 109 target areas. Right panel: the number of fiber samples that reached a certain target area B from seed area A divided by all fiber samples propagated from A provided the connection probability between A and B. The whole set of connection probabilities of seed area A provided its connectional fingerprint that was visualized as a radial graph. Here, we show the simplified finger prints of three nodes with five target areas each. The distance from the center represented the connection probability. After comparing the similarity of connectional fingerprints node by node, we performed post hoc tests to identify abnormal connection probabilities that lead abnormal connectional fingerprints. In this example, the connection probability of node two to its target area $\mathrm{E}$ was reduced in schizophrenia patients compared to controls.

\section{Identifying Brain Regions with Abnormal Connectional Fingerprint}

We identified nodes with abnormal connectional fingerprints through permutation testing of pairwise similarity between any two participants for a certain node. We computed the sum of the absolute difference between two connectional fingerprints and transformed it to a similarity measure using a simple monotonically decreasing exponential function. The similarity of the $k$ th node between the $i$ th subject and the $j$ th subject is:

$$
s_{i j}^{k}=\mathrm{e}^{-d_{i j}^{k} / \alpha} \text { where } d_{i j}^{k}=\left|w_{i}^{k}-w_{j}^{k}\right|
$$

where $w_{i}^{k}$ is the connectional fingerprint of the $i$ th subject from the $k$ th node, $|\cdot|$ represents a sum of all elements' absolute value, and $\alpha$ is a regularization factor, which was set to the half of the maximum difference over all nodes and subjects.

For each node, we computed the pairwise similarity of the corresponding connectional fingerprints for all subjects in both groups ( $n \times m$ similarity matrix, where $n$ is the total number of subjects and $m$ is the number of nodes) and the significance level defining a group difference was obtained through permutation testing under the assumption that the average within-group similarity is larger than the average betweengroup similarity (51) for a connectional fingerprint that differs significantly between groups. To estimate the significance level, we constructed a null distribution of the representative statistic with permutations of group assignment, where the representative statistic is the difference between average within-group similarity and average between-group similarity. Specifically, we computed the value for $N-1$ randomly permuted group assignments, where the significance level was estimated as the number of values, which is equal to or larger than the value from the original group assignment divided by $N$, where $N$ is the number of permutations. A multiple comparison correction for nodes was obtained through the false discovery rate (FDR) procedure (52). We note that this method can be applied to multi-group comparison. We evaluated the suggested statistical test over synthetic data in the Supplementary Material (See SI text 2, Figures S1, S2, and Table S2 in Supplementary Material).

\section{Post hoc Tests}

To identify the connection probabilities that led to the betweengroup differences in the connectional fingerprints, we then compared the connection probabilities of the corresponding fingerprints between groups using permutation testing $(12,53)$ and FDR. Since the connection probabilities of the connectional fingerprint of a certain node are independent from that of another node, multiple comparison correction can be performed separately for each identified node. We note that the connection probability between two areas has to be analyzed in the context of the whole connectional fingerprint of a node. 


\section{Correlation with Clinical Symptoms}

Pearson correlation coefficients were computed between the PANSS-factors (positive, negative, cognitive, depression, excitement, and disorganization), individual PANSS-items and connection probabilities for those nodes with abnormal connectional fingerprints.

\section{Statistics and Visualization}

All topological and statistical operations were conducted using Matlab (version 2009b, Mathworks, Natick, MA, USA). Statistical testing was performed with a permutation test $(50,000$ permutations) (53) and FDR procedure (54) for multiple comparison correction with a threshold of $q=0.05$. A permutation test was employed because the connectivity data were not normally distributed. We implemented the proposed method using Matlab, and uploaded both the codes and the test data set at http://cheolhan.dothome.co.kr/software/.

For visualization routines, we used our in-house code, which draws brain regions and connection probabilities overlapped with the transparent brain. Coordinates of each region were retrieved from the FSL package by calculating the center of mass. As the connectional fingerprint is a node-specific parameter, we used radial graphs (23) to visualize the distribution of each brain region's connectional fingerprint or bar graphs. In a radial graph, the distance represented the magnitude of each connection probability from the graph's center.

\section{RESULTS}

\section{Participants}

We recruited 16 medicated schizophrenia patients [mean age: 34.2 $6 \pm 9.80$ SD, age range: (21-52)] and 17 age- and gender-matched healthy controls [mean age: $32.31 \pm 9.79$, age range: $(19,51)$ ] All patients were on medication at the time of testing receiving atypical antipsychotics. Both groups did not differ significantly in age (permutation test, $p=0.82)$, gender $(p=0.60)$, handedness $(p=0.81)$, and years of education $(p=0.41)$. There was a statistical trend for a difference in verbal intelligence $(p=0.06)$ between groups. Compared to controls, schizophrenia patients achieved lower values in the total score of the BACS battery $(p=0.00001)$ and in the following subtests: verbal memory $(p=0.0004)$, digit sequencing $(p=0.0001)$, verbal fluency $(p=0.00001)$, and symbol coding ( $p=0.0044)$ (Table 1).

\section{Abnormal Connectional Fingerprints in Schizophrenia}

Four abnormal nodes were identified in schizophrenia patients: right medial frontal gyrus (MFG), left putamen (Puta) and the opercular part of the left and right inferior frontal gyrus (IFG.po) (Figure 2) (FDR-corrected, $p<0.05$ ).

To identify the abnormal connection probabilities within each connectional fingerprint, we performed post hoc tests (Table 2). The significance level of connection probabilities using connectional fingerprint diagrams is illustrated in Figure $\mathbf{2}$ and Figures S3-S6 in Supplementary Material. As summarized in Table 2, except for findings in the connectional fingerprints of MFG.r,
TABLE 1 | Demographic data.

\begin{tabular}{|c|c|c|c|}
\hline & Controls $(N=17)$ & Patients $(N=16)$ & $p$-values* \\
\hline Gender & Male $=10$ & Male $=11$ & 0.60 \\
\hline Age & $32.3 \pm 9.8^{a}$ & $34.3 \pm 9.8$ & 0.82 \\
\hline Education (years) & $15.0 \pm 3.8$ & $13.9 \pm 3.0$ & 0.41 \\
\hline Verbal IQ & $29.9 \pm 2.8$ & $28.9 \pm 3.4$ & 0.06 \\
\hline Handedness & $68.8 \pm 25.8$ & $70.5 \pm 23.4$ & 0.81 \\
\hline \multicolumn{4}{|l|}{ PANSS } \\
\hline Positive & - & $11.7 \pm 4.6$ & - \\
\hline Negative & - & $16.9 \pm 6.8$ & - \\
\hline Cognitive & - & $11.4 \pm 4.0$ & - \\
\hline Excitement & - & $5.6 \pm 1.7$ & - \\
\hline Depressive & - & $13.6 \pm 3.3$ & - \\
\hline Total Score & - & $68.3 \pm 17.7$ & - \\
\hline \multicolumn{4}{|l|}{ BACS } \\
\hline Verbal memory & $54.5 \pm 5.9$ & $40.19 \pm 13.5$ & 0.0004 \\
\hline Digit Sequencing & $25.9 \pm 3.3$ & $20.06 \pm 4.6$ & 0.00001 \\
\hline Motor speed & $77.7 \pm 32.2$ & $68.94 \pm 22.9$ & 0.2088 \\
\hline Verbal fluency & $60.9 \pm 12.5$ & $40.12 \pm 15.5$ & 0.00001 \\
\hline Symbol coding & $19.7 \pm 5.9$ & $12.47 \pm 5.3$ & 0.0044 \\
\hline $\begin{array}{l}\text { Reasoning and } \\
\text { problem solving }\end{array}$ & $58.5 \pm 11.3$ & $47.71 \pm 12.4$ & 0.0872 \\
\hline Total score & $421.1 \pm 55.4$ & $326.69 \pm 69.1$ & 0.00001 \\
\hline
\end{tabular}

${ }^{a}$ Mean $\pm S D$.

*Significant values are shown in bold.

all the other significantly different connection probabilities were higher in schizophrenia patients compared to controls relative to the other connection probabilities in the corresponding connectional fingerprints. In the connectional fingerprints of MFG.r, we found reduced connection probabilities to the right Frontal Operculum Cortex (FOpC.r), the right Frontal Orbital Cortex (FOC.r), the right Temporal Pole (TP.r), and the right Central Opercular Cortex (COpC.r). In the connectional fingerprint of IFG.po.r, the connection probability to the right superior frontal gyrus (SFG.r) was increased in schizophrenia patients compared to controls. In the connectional fingerprint of IFG.po.l, we found increased connection probabilities to both the superior frontal gyrus (SFG) and the right paracingulate gyrus (ParaciG.r). In the connectional fingerprint of Puta.l, schizophrenia patients showed a higher connection probability to the right pallidum (Pal.r).

\section{Correlation of Abnormal Projections with Clinical Symptoms}

We calculated the Pearson correlation between PANSS-factors and the connection probabilities that showed significant group differences. While no significant correlations between the main factors of the PANSS and the connection probabilities were detected, we found the following significant correlations with individual PANSS-items: in the connectional fingerprint of the MFG.r, the connection probability with COpC.r was correlated with "lack of spontaneity" $(p=0.0048, r=0.7062)$, "blunted affect" ( $p=0.0133, r=0.6420)$ and "poor attention", the connection probability with FOC.r was correlated with "depression" ( $p=0.0414, r=-0.5503)$. In the connectional fingerprint of the Puta.r, the connection probability with Pal.r correlated with 


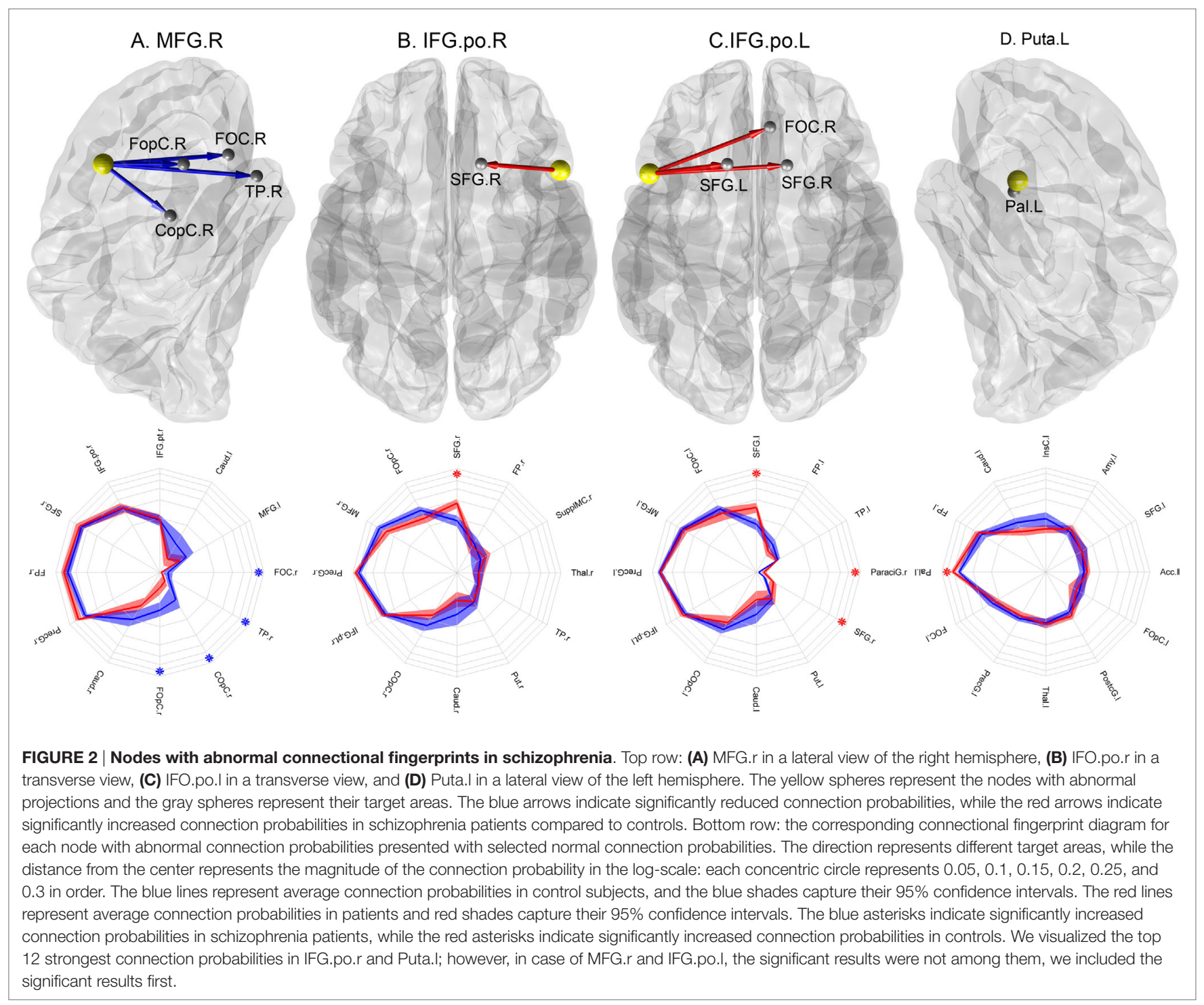

"disorientation" ( $p=0.0407, r=0.5520)$. In the connectional fingerprint of the IFG.po.r, the connection probability with SFG.r correlated with "disorientation" ( $p=0.0202, r=0.6111)$ and "depression" ( $p=0.0107, r=0.6569)$. These results did not survive multiple comparison correction, however. No significant correlation with BACS scores was observed.

\section{DISCUSSION}

\section{Abnormal Connectional Fingerprints in Schizophrenia}

Our study provides the first whole-brain analysis of connectional fingerprints in a clinical sample. Through connectional fingerprint analysis of DTI-data, we provide a complementary view of connectivity changes in schizophrenia that is consistent with the "dysconnection" hypothesis (34). The dysconnection hypothesis suggests that schizophrenia is not only characterized by reduced connectivity but also involves hyperconnected areas in several networks, such as the cortico-subcortical pathways $(55,56)$, the default-mode network $(18,57)$, and the language processing network $(58,59)$.

Our data suggest connectivity abnormalities in several nodes of the frontal cortex, a brain region that has been traditionally implicated in the pathophysiology of schizophrenia (60). Specifically, our findings in the MFG and the inferior frontal gyrus (IFG) are consistent with graph theoretical studies that have indicated abnormal organization and connectivity of frontal networks (10, 11). Functional studies suggest that schizophrenia patients show hyperconnectivity of frontal areas during rest (18). In addition, aberrant modulation of frontal activity has been implicated in a number of cognitive dysfunctions in schizophrenia, including working memory (61) as well as impaired social communication $(62,63)$ and self-other distinction (64). 
TABLE 2 | Post hoc tests of abnormal connectional fingerprints.

\begin{tabular}{llcc}
\hline $\begin{array}{l}\text { Nodes with abnormal } \\
\text { connectional fingerprints }\end{array}$ & $\begin{array}{l}\text { Target } \\
\text { area }^{\mathbf{a}}\end{array}$ & $\begin{array}{c}\text { Pseudo } \\
\text {-statistics }^{\mathbf{b}}\end{array}$ & $\begin{array}{c}\text { Significance } \\
\text { level (FDR } \\
\text { adjusted) }\end{array}$ \\
\hline MFG.r & FOpC.r & 3.2388 & $p=0.0391$ \\
& FOC.r & 2.1950 & $p=0.0391$ \\
& TP.r & 2.7085 & $p=0.0391$ \\
& COpC.r & 2.4573 & $p=0.0391$ \\
IFG.po.r & SFG.r & $\mathbf{- 3 . 6 2 8 4}$ & $p=0.0066$ \\
IFG.po.I & SFG.I & $\mathbf{- 3 . 4 5 4 2}$ & $p=0.0110$ \\
& SFG.r & $\mathbf{- 3 . 2 6 4 2}$ & $p=0.0110$ \\
& ParaciG.r & $\mathbf{- 2 . 9 1 7 8}$ & $p=0.0117$ \\
Puta.l & Pal.l & $-\mathbf{3 . 0 7 9 2}$ & $p=0.0484$
\end{tabular}

Permutation test, FDR adjusted. See Table S1 in Supplementary Material for abbreviations.

aTarget area of abnormal connection probability within the abnormal connectional fingerprint.

'Controls' mean-patients' mean, normalized by mean and SD over permutations, thus, negative pseudo t-statistics represents increased connection in patients (shown in bold).

A second brain region characterized by abnormal connectional fingerprints was the putamen. Striatal circuits and their interactions with cortical regions, in particular with frontal areas, are crucially involved in working memory (65) as well as in reward processing during normal brain functioning (66). Functional and anatomical studies in schizophrenia have provided evidence for impaired striatal-cortical processes underlying these processes (67-69), suggesting that abnormal, striatal connectional fingerprints could contribute toward behavioral abnormalities in schizophrenia. This hypothesis is furthermore supported by the fact that in the connectional fingerprint of the MFG the connection probabilities to the left and right Caudate showed reduced values in schizophrenia patients albeit an uncorrected significance level (see Figure S1 in Supplementary Material).

Possible reasons for connectivity anomalies in schizophrenia may be aberrant myelination (70) and pruning during different developmental stages (71) that impair normal brain maturation with segregation of anatomically close areas and integration of distributed functionally specialized areas (72). In addition, changes in connectivity may also be due to the prolonged presence of positive symptoms that could lead to changes in the architecture of the cortical network as a result of use-dependent strengthening of cortico-cortical connections (73).

\section{Methodological Issues}

Investigations into the organization of anatomical networks remain challenging in clinical populations because of abnormalities in GM/WM-volume that can confound streamline-numbers detected through tractography algorithms, for example, as these are influenced by overall WM connectivity, and the volume of the seed area in GM. Accordingly, edge comparison analysis based on the number of streamlines or fiber tracts (absolute connectivity) might not be sufficient.
The use of the connectional fingerprint has several potential advantages: first, connection probabilities of connectional fingerprints are independent from the overall connectivity (22), because they are normalized for all fibers of the seed area. Second, connectional fingerprints are also independent from the seed volume and, respectively, the total brain volume (33), because each pattern is first calculated for each voxel and subsequently averaged for the whole seed area. Hence, we suggest that a connectional fingerprint is suitable to investigate the "local" or node-specific connectivity pattern more precisely $(33,74)$ compared to approaches involving the normalization of connectivity between each pair of nodes by the total number of fiber tracts (75).

However, our methodological approach has a several limitations that need to be considered in future studies. First, it is important to note that the selection of the similarity measure determines the sensitivity of the tests and alternative measures have to be studied in future studies (See text 3 in Supplementary Material). Second, probabilistic tractography is sensitive to the distance bias. Hence, if there were systematic differences in the distances between two brain regions in patients compared to healthy subjects, the connectional fingerprints would differ between groups. Thus, our result should be carefully interpreted.

It is important to note that significant difference in the connectional fingerprints between groups is detected if either few connection probabilities within a connectional fingerprint differ between groups by a large magnitude or many connectional probabilities within a connectional fingerprint differ by a small magnitude. This was also confirmed by the analysis of synthetic data (See text 2 in Supplementary Material). For the post hoc tests of MFG.r and IFG.po, we observed significant differences in very small mean connection probabilities. However, small connection probabilities may result from crossing fibers and long distances between seed and target areas. Hence, changes in the tail of the connectional fingerprint need to be interpreted with care. However, it should be emphasized that connection probabilities that showed relative hyperconnectivity in patients only involved paths with strong mean connection probabilities in the connectional fingerprint (from IFG.po.r to SFG.r; from IFG.po.l to SFG.l, and from Puta.1 to Pal.1, See Figure 2).

Another limitation of our study is the relatively small sample size. However, our results of abnormal connectional fingerprints (Figure 2) and connection probabilities (Figures S3-S6 in Supplementary Material) were significant after the multiple comparison correction. In addition, we would like to note that we did not acquire EPI distortion field maps and did not perform EPI distortion field correction. Thus, the registration can be inaccurate and we may have inaccurate tractography results in regions with high EPI distortion. However, we visually validated the registration to account for this. In addition, we cannot exclude the possibility that some of the changes observed in connectional fingerprints are due to effects associated with antipsychoticmedication treatment. In particular, abnormalities observed in the putamen could be influenced by antipsychotic medication as 
this region rich in D2 receptors that are targets for antipsychotic medication (76).

\section{CONCLUSION}

In summary, we present a new approach to systematically investigate local connectivity independent of overall GM/WM changes of seed areas through connectional fingerprint analysis. This approach provides novel evidence for an altered organization of the connectome in schizophrenia that implicates changes in the network architecture of WM pathways as a core anatomical deficit. Specifically, we demonstrate altered connectional fingerprints in frontal and subcortical areas involving both hypo- and hyperconnectivity relatively to the other connection probabilities in their corresponding connectional fingerprints. Future studies need to address the functional consequences of abnormal connectional fingerprints. Since our findings are in line with previous graph theoretical analysis of fMRI data (13), we suggest that connectional fingerprint analysis is a useful parameter approach in multimodal studies that investigate the link between functional and structural data. This hypothesis is strengthened by previous studies that demonstrated high correspondence between parcelation based on the connectional fingerprints and segregation based on task-specific fMRI data $(29,31)$.

\section{REFERENCES}

1. Bullmore E, Sporns O. Complex brain networks: graph theoretical analysis of structural and functional systems. Nat Rev Neurosci (2009) 10:186-98. doi:10.1038/nrn2575

2. Hagmann P, Kurant M, Gigandet X, Thiran P, Wedeen VJ, Meuli R, et al. Mapping human whole-brain structural networks with diffusion MRI. PLoS One (2007) 2:e597. doi:10.1371/journal.pone.0000597

3. Hagmann P, Cammoun L, Gigandet X, Meuli R, Honey CJ, Wedeen VJ, et al. Mapping the structural core of human cerebral cortex. PLoS Biol (2008) 6:e159. doi:10.1371/journal.pbio.0060159

4. Iturria-Medina Y, Sotero RC, Canales-Rodriguez EJ, Aleman-Gomez Y, Melie-Garcia L. Studying the human brain anatomical network via diffusion-weighted MRI and Graph Theory. Neuroimage (2008) 40:1064-76. doi:10.1016/j.neuroimage.2007.10.060

5. Anderson A, Cohen MS. Decreased small-world functional network connectivity and clustering across resting state networks in schizophrenia: an fMRI classification tutorial. Front Hum Neurosci (2013) 7:520. doi:10.3389/ fnhum.2013.00520

6. Rubinov M, Bullmore E. Schizophrenia and abnormal brain network hubs. Dialogues Clin Neurosci (2013) 15:339-49.

7. Shim M, Kim DW, Lee SH, Im CH. Disruptions in small-world cortical functional connectivity network during an auditory oddball paradigm task in patients with schizophrenia. Schizophr Res (2014) 156:197-203. doi:10.1016/j. schres.2014.04.012

8. Friston K. Disconnection and cognitive dysmetria in schizophrenia. Am J Psychiatry (2005) 162:429-32. doi:10.1176/appi.ajp.162.3.429

9. Uhlhaas PJ, Singer W. Neuronal dynamics and neuropsychiatric disorders: toward a translational paradigm for dysfunctional large-scale networks. Neuron (2012) 75:963-80. doi:10.1016/j.neuron.2012.09.004

10. Bassett DS, Bullmore E, Verchinski BA, Mattay VS, Weinberger DR, Meyer-Lindenberg A. Hierarchical organization of human cortical networks in health and schizophrenia. JNeurosci (2008) 28:9239-48. doi:10.1523/ JNEUROSCI.1929-08.2008

\section{AUTHOR CONTRIBUTIONS}

SET, CEH, MK, PJU designed the research and wrote the manuscript. SET collected the data. SET, CEH, ARJ preprocessed the data. SET, CEH developed the method and analyzed the data. WS, RD, KM contributed towards manuscript preparation with interpretation.

\section{FUNDING}

$\mathrm{CEH}$ was funded by National Research Foundation of Korea funded by the Ministry of Education, Science and Technology (MSIP-R1A1A1008173). MK was funded by National Research Foundation of Korea funded by the Ministry of Education, Science and Technology (R32-10142), the Royal Society (RG/2006/R2), the CARMEN e-science project (http://www.carmen.org.uk) funded by EPSRC (EP/E002331/1), and the Human Green Brain Project (http://www.greenbrainproject.org) funded by EPSRC (EP/K026992/1). PU, SET, and WS were supported by the MaxPlanck Society. None of authors has financial conflicts.

\section{SUPPLEMENTARY MATERIAL}

The Supplementary Material for this article can be found online at http://journal.frontiersin.org/article/10.3389/fpsyt.2016.00114

11. van den Heuvel MP, Mandl RC, Stam CJ, Kahn RS, Hulshoff Pol HE Aberrant frontal and temporal complex network structure in schizophrenia: a graph theoretical analysis. JNeurosci (2010) 30:15915-26. doi:10.1523/ JNEUROSCI.2874-10.2010

12. Zalesky A, Fornito A, Seal ML, Cocchi L, Westin C-F, Bullmore ET, et al. Disrupted axonal fiber connectivity in schizophrenia. Biol Psychiatry (2011) 69:80-9. doi:10.1016/j.biopsych.2010.08.022

13. Skudlarski P, Jagannathan K, Anderson K, Stevens MC, Calhoun VD, Skudlarska BA, et al. Brain connectivity is not only lower but different in schizophrenia: a combined anatomical and functional approach. Biol Psychiatry (2010) 68:61-9. doi:10.1016/j.biopsych.2010.03.035

14. Zalesky A, Fornito A, Egan GF, Pantelis C, Bullmore ET. The relationship between regional and inter-regional functional connectivity deficits in schizophrenia. Hum Brain Mapp (2012) 33:2535-49. doi:10.1002/hbm.21379

15. Wang Q, Su TP, Zhou Y, Chou KH, Chen IY, Jiang T, et al. Anatomical insights into disrupted small-world networks in schizophrenia. Neuroimage (2012) 59:1085-93. doi:10.1016/j.neuroimage.2011.09.035

16. Anticevic A, Hu X, Xiao Y, Hu J, Li F, Bi F, et al. Early-course unmedicated schizophrenia patients exhibit elevated prefrontal connectivity associated with longitudinal change. JNeurosci (2015) 35:267-86. doi:10.1523/ JNEUROSCI.2310-14.2015

17. Cui LB, Liu J, Wang LX, Li C, Xi YB, Guo F, et al. Anterior cingulate cortex-related connectivity in first-episode schizophrenia: a spectral dynamic causal modeling study with functional magnetic resonance imaging. Front Hum Neurosci (2015) 9:589. doi:10.3389/fnhum.2015.00589

18. Whitfield-Gabrieli S, Thermenos H, Milanovic S, Tsuang M, Faraone S, Mccarley R, et al. Hyperactivity and hyperconnectivity of the default network in schizophrena and first-degree relatives of persons with schizoprhenia. Proc Natl Acad Sci U S A (2009) 106(4):1279-84. doi:10.1073/pnas. 0809141106

19. Colibazzi T, Wexler BE, Bansal R, Hao X, Liu J, Sanchez-Pena J, et al. Anatomical abnormalities in gray and white matter of the cortical surface in persons with schizophrenia. PLoS One (2013) 8:e55783. doi:10.1371/journal. pone. 0055783 
20. Sprooten E, Fleming KM, Thomson PA, Bastin ME, Whalley HC, Hall J, et al. White matter integrity as an intermediate phenotype: exploratory genomewide association analysis in individuals at high risk of bipolar disorder. Psychiatry Res (2013) 206:223-31. doi:10.1016/j.psychres.2012.11.002

21. Thormodsen R, Rimol LM, Tamnes CK, Juuhl-Langseth M, Holmen A, Emblem KE, et al. Age-related cortical thickness differences in adolescents with early-onset schizophrenia compared with healthy adolescents. Psychiatry Res (2013) 214:190-6. doi:10.1016/j.pscychresns.2013.07.003

22. Croxson PL, Johansen-Berg H, Behrens TE, Robson MD, Pinsk MA, Gross CG, et al. Quantitative investigation of connections of the prefrontal cortex in the human and macaque using probabilistic diffusion tractography. JNeurosci (2005) 25:8854-66. doi:10.1523/JNEUROSCI.1311-05.2005

23. Passingham RE, Stephan KE, Kotter R. The anatomical basis of functional localization in the cortex. Nat Rev Neurosci (2002) 3:606-16. doi:10.1038/ nrn893

24. Behrens TE, Johansen-Berg H, Woolrich MW, Smith SM, WheelerKingshott CA, Boulby PA, et al. Non-invasive mapping of connections between human thalamus and cortex using diffusion imaging. Nat Neurosci (2003) 6:750-7. doi:10.1038/nn1075

25. Finn ES, Shen X, Scheinost D, Rosenberg MD, Huang J, Chun MM, et al. Functional connectome fingerprinting: identifying individuals using patterns of brain connectivity. Nat Neurosci (2015) 18:1664-71. doi:10.1038/ nn. 4135

26. Johansen-Berg H, Behrens TE, Robson MD, Drobnjak I, Rushworth MF, Brady JM, et al. Changes in connectivity profiles define functionally distinct regions in human medial frontal cortex. Proc Natl Acad Sci U S A (2004) 101:13335-40. doi:10.1073/pnas.0403743101

27. Devlin JT, Sillery EL, Hall DA, Hobden P, Behrens TE, Nunes RG, et al. Reliable identification of the auditory thalamus using multi-modal structural analyses. Neuroimage (2006) 30:1112-20. doi:10.1016/j.neuroimage.2005.11.025

28. Anwander A, Tittgemeyer M, Von Cramon DY, Friederici AD, Knosche TR. Connectivity-based parcellation of Broca's area. Cereb Cortex (2007) 17:816-25. doi:10.1093/cercor/bhk034

29. Klein JC, Behrens TE, Robson MD, Mackay CE, Higham DJ, JohansenBerg H. Connectivity-based parcellation of human cortex using diffusion MRI: establishing reproducibility, validity and observer independence in BA 44/45 and SMA/pre-SMA. Neuroimage (2007) 34:204-11. doi:10.1016/j. neuroimage.2006.08.022

30. Tomassini V, Jbabdi S, Klein JC, Behrens TE, Pozzilli C, Matthews PM, et al. Diffusion-weighted imaging tractography-based parcellation of the human lateral premotor cortex identifies dorsal and ventral subregions with anatomical and functional specializations. J Neurosci (2007) 27:10259-69. doi:10.1523/JNEUROSCI.2144-07.2007

31. Johansen-Berg H, Behrens TE, Sillery E, Ciccarelli O, Thompson AJ, Smith SM, et al. Functional-anatomical validation and individual variation of diffusion tractography-based segmentation of the human thalamus. Cereb Cortex (2005) 15:31-9. doi:10.1093/cercor/bhh105

32. Gong G, Rosa-Neto P, Carbonell F, Chen ZJ, He Y, Evans AC. Age- and gender-related differences in the cortical anatomical network. J Neurosci (2009) 29:15684-93. doi:10.1523/JNEUROSCI.2308-09.2009

33. Duarte-Carvajalino JM, Jahanshad N, Lenglet C, Mcmahon KL, De Zubicaray GI, Martin NG, et al. Hierarchical topological network analysis of anatomical human brain connectivity and differences related to sex and kinship. Neuroimage (2012) 59(4):3784-804. doi:10.1016/j.neuroimage.2011. 10.096

34. Stephan KE, Baldeweg T, Friston KJ. Synaptic plasticity and dysconnection in schizophrenia. Biol Psychiatry (2006) 59:929-39. doi:10.1016/j. biopsych.2005.10.005

35. Oldfield R. The assessment and analysis of handedness: the Edinburgh inventory. Neuropsychologia (1971) 9(1):97-113.

36. Keefe R, Goldberg T, Harvey P, Gold J, Poe M, Coughenour L. The brief assessment of cognition in schizophrenia: reliability, sensitivity, and comparison with a standard neurocognitive battery. Schizophr Res (2004) 68(2-3):283-97. doi:10.1016/j.schres.2003.09.011

37. Lehrl M. Der Mehfach-Wortwahl-Test. Weinheim: Beltz Test Verlag (1985).

38. Kay S, Opler L, Fiszbein A. Significance of positive and negative syndromes in chronic schizophrenia. Br J Psychiatry (1986) 149:439-48. doi:10.1192/ bjp.149.4.439
39. Lindenmayer JP, Bernstein-Hyman R, Grochowski S, Bark N. Psychopathology of schizophrenia: initial validation of a 5-factor model. Psychopathology (1995) 28(1):22-31. doi:10.1159/000284896

40. Cuesta M, Peralta V. Psychopathological dimensions in schizophrenia. Schizophr Bull (1995) 21(3):473-82. doi:10.1093/schbul/21.3.473

41. Smith S, Jenkinson M, Woolrich M, Beckmann C, Behrens T, JohansenBerg $\mathrm{H}$, et al. Advances in functional and structural MR image analysis and implementation as FSL. Neuroimage (2004) 23(Suppl 1):S208-19. doi:10.1016/j.neuroimage.2004.07.051

42. Jenkinson M, Bannister S, Brady J, Smith S. Improved optimisation for the robust and accurate linear registration and motion correction of brain images. Neuroimage (2002) 17(2):825-41. doi:10.1006/nimg.2002.1132

43. Fox M, Synder A, Vincent J, Corbetta M, Van Essen D, Reichle M. The human brain is intrinsically organized into dynamic, anticorrelated functional networks. Proc Natl Acad Sci U S A (2005) 102(27):9673-8. doi:10.1073/ pnas.0504136102

44. Behrens T, Johansen-Berg H, Jbabdi S, Rushworth M, Woolrich M. Probabilistic diffusion tractography with multiple fibre orientations. What can we gain? Neuroimage (2007) 34(1):144-55. doi:10.1016/j.neuroimage.2006.09.018

45. Mori S, Barker PB. Diffusion magnetic resonance imaging: its principle and applications. Anat Rec (1999) 257:102-9. doi:10.1002/ (SICI)1097-0185(19990615)257:3<102::AID-AR7>3.0.CO;2-6

46. Jones DK, Knosche TR, Turner R. White matter integrity, fiber count, and other fallacies: the do's and don'ts of diffusion MRI. Neuroimage (2013) 73:239-54. doi:10.1016/j.neuroimage.2012.06.081

47. Jones D. Studying connections in the living human brain with diffusion MRI. Cortex (2008) 44(8):936-52. doi:10.1016/j.cortex.2008.05.002

48. Heiervang E, Behrens TE, Mackay CE, Robson MD, Johansen-Berg H. Between session reproducibility and between subject variability of diffusion MR and tractography measures. Neuroimage (2006) 33:867-77. doi:10.1016/j. neuroimage.2006.07.037

49. Owen JP, Ziv E, Bukshpun P, Pojman N, Wakahiro M, Berman JI, et al. Testretest reliability of computational network measurements derived from the structural connectome of the human brain. Brain Connect (2013) 3:160-76. doi:10.1089/brain.2012.0121

50. Echtermeyer C, Han CE, Rotarska-Jagiela A, Mohr H, Uhlhaas PJ, Kaiser M. Integrating temporal and spatial scales: human structural network motifs across age and region of interest size. Front Neuroinform (2011) 5:10. doi:10.3389/fninf.2011.00010

51. Kropf S, Heuer H, Gruning M, Smalla K. Significance test for comparing complex microbial community fingerprints using pairwise similarity measures. J Microbiol Methods (2004) 57:187-95. doi:10.1016/j. mimet.2004.01.002

52. Benjamini $Y$, Hochberg Y. Controlling the false discovery rate: a practical and powerful approach to multiple testing. J R Stat Soc Series B Stat Methodol (1995) 57(1):289-300.

53. Nichols TE, Holmes AP. Nonparametric permutation tests for functional neuroimaging: a primer with examples. Hum Brain Mapp (2002) 15:1-25. doi:10.1002/hbm.1058

54. Genovese CR, Lazar NA, Nichols T. Thresholding of statistical maps in functional neuroimaging using the false discovery rate. Neuroimage (2002) 15:870-8. doi:10.1006/nimg.2001.1037

55. Salvador R, Sarro S, Gomar JJ, Ortiz-Gil J, Vila F, Capdevila A, et al. Overall brain connectivity maps show cortico-subcortical abnormalities in schizophrenia. Hum Brain Mapp (2010) 31:2003-14. doi:10.1002/hbm.20993

56. Zhang D, Guo L, Hu X, Li K, Zhao Q, Liu T. Increased cortico-subcortical functional connectivity in schizophrenia. Brain Imaging Behav (2012) 6(1):27-35. doi:10.1007/s11682-011-9138-z

57. Zhou Y, Liang M, Tian L, Wang K, Hao Y, Liu H, et al. Functional disintegration in paranoid schizophrenia using resting-state fMRI. Schizophr Res (2007) 97:194-205. doi:10.1016/j.schres.2007.05.029

58. Hubl D, Koenig T, Strik W, Federspiel A, Kreis R, Boesch C, et al. Pathways that make voices: white matter changes in auditory hallucinations. Arch Gen Psychiatry (2004) 61:658-68. doi:10.1001/archpsyc.61.7.658

59. Szycik GR, Ye Z, Mohammadi B, Dillo W, Te Wildt BT, Samii A, et al. Maladaptive connectivity of Broca's area in schizophrenia during audiovisual speech perception: an fMRI study. Neuroscience (2013) 253:274-82. doi:10.1016/j.neuroscience.2013.08.041 
60. Weinberger DR, Berman KF, Suddath R, Torrey EF. Evidence of dysfunction of a prefrontal-limbic network in schizophrenia: a magnetic resonance imaging and regional cerebral blood flow study of discordant monozygotic twins. Am J Psychiatry (1992) 149:890-7. doi:10.1176/ajp.149.7.890

61. Cannon TD, Glahn DC, Kim J, Van Erp TG, Karlsgodt K, Cohen MS, et al. Dorsolateral prefrontal cortex activity during maintenance and manipulation of information in working memory in patients with schizophrenia. Arch Gen Psychiatry (2005) 62(10):1071-80. doi:10.1001/archpsyc.62.10.1071

62. Park IH, Park HJ, Chun JW, Kim EY, Kim JJ. Prefrontal functional dissociation in the semantic network of patients with schizophrenia. Neuroreport (2008) 19:1391-5. doi:10.1097/WNR.0b013e32830cebff

63. Yamasaki S, Yamasue H, Abe O, Suga M, Yamada H, Inoue H, et al. Reduced gray matter volume of pars opercularis is associated with impaired social communication in high-functioning autism spectrum disorders. Biol Psychiatry (2010) 68:1141-7. doi:10.1016/j.biopsych.2010.07.012

64. Backasch B, Sommer J, Klohn-Saghatolislam F, Muller MJ, Kircher TT, Leube DT. Dysconnectivity of the inferior frontal gyrus: implications for an impaired self-other distinction in patients with schizophrenia. Psychiatry Res (2014) 223:202-9. doi:10.1016/j.pscychresns.2014.05.007

65. Baier B, Karnath HO, Dieterich M, Birklein F, Heinze C, Muller NG. Keeping memory clear and stable - the contribution of human basal ganglia and prefrontal cortex to working memory. J Neurosci (2010) 30:9788-92. doi:10.1523/ JNEUROSCI.1513-10.2010

66. Staudinger MR, Erk S, Walter H. Dorsolateral prefrontal cortex modulates striatal reward encoding during reappraisal of reward anticipation. Cereb Cortex (2011) 21:2578-88. doi:10.1093/cercor/bhr041

67. Quide Y, Morris RW, Shepherd AM, Rowland JE, Green MJ. Task-related fronto-striatal functional connectivity during working memory performance in schizophrenia. Schizophr Res (2013) 150:468-75. doi:10.1016/j. schres.2013.08.009

68. Grimm O, Heinz A, Walter H, Kirsch P, Erk S, Haddad L, et al. Striatal response to reward anticipation: evidence for a systems-level intermediate phenotype for schizophrenia. JAMA Psychiatry (2014) 71:531-9. doi:10.1001/ jamapsychiatry.2014.9

69. Womer FY, Wang L, Alpert KI, Smith MJ, Csernansky JG, Barch DM, et al. Basal ganglia and thalamic morphology in schizophrenia and bipolar disorder. Psychiatry Res (2014) 223:75-83. doi:10.1016/j.pscychresns.2014.05.017
70. Davis KL, Stewart DG, Friedman JI, Buchsbaum M, Harvey PD, Hof PR, et al. White matter changes in schizophrenia: evidence for myelin-related dysfunction. Arch Gen Psychiatry (2003) 60:443-56. doi:10.1001/archpsyc.60.5.443

71. Bartzokis G. Schizophrenia: breakdown in the well-regulated lifelong process of brain development and maturation. Neuropsychopharmacology (2002) 27:672-83. doi:10.1016/S0893-133X(02)00364-0

72. Fair DA, Cohen AL, Power JD, Dosenbach NU, Church JA, Miezin FM, et al. Functional brain networks develop from a "local to distributed" organization. PLoS Comput Biol (2009) 5:e1000381. doi:10.1371/journal.pcbi.1000381

73. Balu DT, Coyle JT. Neuroplasticity signaling pathways linked to the pathophysiology of schizophrenia. Neurosci Biobehav Rev (2011) 35:848-70. doi:10.1016/j.neubiorev.2010.10.005

74. Costafreda S, Fu C, Picchioni M, Touloupoulou T, Mcdonald C, Walshe M, et al. Pattern of neural responses to verbal fluency shows diagnostic specificity for schizophrenia and bipolar disorder. BMC Psychiatry (2011) 11:18. doi:10.1186/1471-244X-11-18

75. Jahanshad N, Aganj I, Lenglet C, Joshi A, Jin Y, Barysheva M, et al. Sex differences in the human connectome: 4-Tesla high angular resolution diffusion imaging (HARDI) tractography in 234 young adult twins. Biomedical Imaging: From Nano to Macro, 2011 IEEE International Symposium. Chigago, IL: IEEE (2011). p. 939-43.

76. Price R, Salavati B, Graff-Guerrero A, Blumberger DM, Mulsant BH, Daskalakis ZJ, et al. Effects of antipsychotic D2 antagonists on long-term potentiation in animals and implications for human studies. Prog Neuropsychopharmacol Biol Psychiatry (2014) 54C:83-91. doi:10.1016/j.pnpbp.2014.05.001

Conflict of Interest Statement: The authors declare that the research was conducted in the absence of any commercial or financial relationships that could be construed as a potential conflict of interest.

Copyright (C) 2016 Edwin Thanarajah, Han, Rotarska-Jagiela, Singer, Deichmann, Maurer, Kaiser and Uhlhaas. This is an open-access article distributed under the terms of the Creative Commons Attribution License (CC BY). The use, distribution or reproduction in other forums is permitted, provided the original author(s) or licensor are credited and that the original publication in this journal is cited, in accordance with accepted academic practice. No use, distribution or reproduction is permitted which does not comply with these terms. 